\title{
Robotic-assisted total mesorectal excision with the single-docking technique for patients with rectal cancer
}

\author{
Ching-Wen Huang ${ }^{1,2}$, Hsiang-Lin Tsai 1,2,4,5, Yung-Sung Yeh ${ }^{2,3,6}$, Wei-Chih Su, ${ }^{2,6}$, Ming-Yii Huang ${ }^{7}$, \\ Chun-Ming Huang ${ }^{7}$, Yu-Tang Chang ${ }^{5,6,8}$ and Jaw-Yuan Wang ${ }^{1,2,5,6,9,10,11^{*}}$ (i)
}

\begin{abstract}
Background: The robotic system has advantages of high-definition three-dimensional vision and articular instruments with high dexterity, allowing more precise dissection in the deep and narrow pelvic cavity.

Methods: We enrolled 95 patients with stage I-III rectal cancer (adenocarcinoma) who underwent totally robotic-assisted total mesorectal excision (TME) with single-docking technique at a single institution between September 2013 and December 2016.

Results: Of the 95 patients, 48 (50.5\%) and 30 (31.6\%) patients had lower and middle rectal cancers, respectively. Of the 75 (78.9\%) patients undergoing preoperative concurrent chemoradiotherapy (CCRT), 27 (28.4\%) exhibited pathologic complete response ( $\mathrm{pCR}$ ). Only four (4.2\%) patients underwent abdominoperineal resection and the sphincter preservation rate was 95.8\%. RO resection was performed in 92 (96.8\%) patients. Circumferential resection margin (CRM) and distal resection margin (DRM) were positive in $2(2.1 \%)$ and $1(1.1 \%)$ patients, respectively. The anastomotic leakage rate was 5.4\% (5/95 patients). The overall complication rate was 17.9\% (17/95 patients); most of them were mild. No 30-day hospital mortality occurred, and no patients required conversion to open surgery. In 92 patients undergoing R0 resection, 2-year overall survival was $94 \%$ and 2 -year disease-free survival was $83 \%$.

Conclusions: The results demonstrated that totally robotic-assisted TME with the single-docking technique is safe and feasible for patients with rectal cancer, with or without preoperative CCRT. Moreover, favorable pCR rate, RO resection rate, CRM, DRM, sphincter preservation rate, and short-term oncological outcomes can be achieved by combining this approach with appropriate preoperative CCRT.
\end{abstract}

Keywords: Robotic-assisted total mesorectal excision, Single-docking, Rectal cancer, R0 resection, Circumferential resection margin

\section{Background}

In the past three decades, several advancements including improvement in surgical techniques and the development of new therapeutic modalities have improved treatment outcomes of rectal cancers. Total mesorectal excision (TME) surgery, which was described by Heald and Ryall [1] in 1982, remarkably improves the clinical

\footnotetext{
* Correspondence: cy614112@ms14.hinet.net; jayuwa@cc.kmu.edu.tw ${ }^{1}$ Graduate Institute of Medicine, College of Medicine, Kaohsiung Medical University, Kaohsiung, Taiwan

${ }^{2}$ Division of Colorectal Surgery, Department of Surgery, Kaohsiung Medical University Hospital, Kaohsiung Medical University, Kaohsiung, Taiwan Full list of author information is available at the end of the article
}

outcomes of patients with rectal cancer; thus it has served as the standard surgical procedure for such patients. A 5-year local recurrence rate of 5\% in patients who undergone TME surgery alone was reported by MacFarlane et al. [2]. In addition, preoperative concurrent chemoradiotherapy (CCRT) considerably helps in improving the local recurrence rate in patients with locally advanced rectal cancer (LARC). A German study reported a considerable decrease in local recurrence in patients receiving preoperative CCRT $[3,4]$. The similar results were also reported by other studies [5-7] and preoperative CCRT has been the recommended treatment for patients with LARC. 
Laparoscopic rectal surgery with TME is still not accepted worldwide as the standard surgical procedure for rectal cancer treatment because it requires highly technically skilled surgeons experienced in minimally invasive surgeries $[8,9]$. The robotic system (da Vinci ${ }^{\oplus}$ Surgical System, Intuitive Surgical, Inc., Sunnyvale, CA) has several advantages such as high-definition three-dimensional vision with up to $10 \times$ magnification, the articulatory instruments of the system, the surgeon-controlled camera platform, and stable traction provided by the robotic arm. Thus, dissection in the confined pelvic cavity can be performed more precisely by using this robotic system. Since the first robotic colon surgery in 2002 [10], the disadvantages of conventional laparoscopic colorectal surgery are expected to be solved by robotic systems. Several studies have reported that compared with conventional laparoscopic and open surgeries for rectal cancers, clinical and short-term oncological outcomes of robotic surgery are more favorable [11-14].

Rectal cancer surgery is a multiquadrant operation involving the left upper quadrant, left lower quadrant, and pelvic cavity. Surgical procedures include dissection of the lymph nodes; ligation of the inferior mesentery artery (IMA) and inferior mesentery vein (IMV); mobilization of the splenic flexure of the colon, descending colon, and sigmoid colon; and dissection of the pelvic. The hybrid technique with laparoscopic dissection of the lymph nodes, ligation of IMA and IMV, mobilization of the colon, and robotic dissection of the pelvic developed first. Thereafter, totally robotic surgeries with the dual-docking technique or single-docking flip-arm technique were performed. Several robotic surgical techniques including hybrid, totally robotic (including dual-docking and singledocking flip-arm techniques), and reverse hybrid are currently being used [15].

In the present study, we present a method of the single-docking technique without moving the robotic surgical cart and repositioning robotic arms to perform totally robotic radical rectal cancer surgery. In addition, we discuss the short-term oncological outcomes of patients with rectal cancer who underwent totally robotic-assisted TME with the single-docking technique.

\section{Methods}

\section{Patients}

We included 95 patients with stage I-III rectal cancer (adenocarcinoma) who underwent totally roboticassisted TME with the single-docking technique with the $\mathrm{da} \mathrm{Vinci}^{\odot}$ surgical system at a single-institution between September 2013 and December 2016. This study was approved by the institutional review board of the Kaohsiung Medical University Hospital (KMUHIRB-E-20150003). Written informed consent to participate was obtained from each patient before performing the robotic surgery.
All patients routinely underwent preoperative colonoscopy and abdominal and pelvic computed tomography (CT) or magnetic resonance imaging (MRI) for preoperative staging. On the basis of the distance from the anal verge, rectal cancer was categorized into upper $(11-15 \mathrm{~cm})$, middle $(6-10 \mathrm{~cm})$, and lower $(\leq 5 \mathrm{~cm})$. Patients with T3, T4, or $\mathrm{N}+$ rectal cancer received preoperative CCRT. Furthermore, the 5-fluorouracil, leucovorin, and oxaliplatin (FOLFOX) regimen was prescribed for patients with T4 or $\mathrm{N}+$ rectal cancer and a fluoropyrimidine-based regimen was prescribed for patients with T3 N0 rectal cancer. Longcourse radiotherapy (LCRT, total 5000 cGy in 25 fractions) was concurrently administered. Totally robotic-assisted TME with the single-docking technique was scheduled after more than 6 weeks after radiotherapy completion.

Clinicopathological features and perioperative parameters or outcomes were collected and evaluated, including age; sex; histological type; tumor, node, and metastasis (TNM classification); perineural invasion; vascular invasion; time interval between completion of preoperative radiotherapy and robotic surgery; tumor location (distance from anal verge); pre-CCRT, preoperative, and postoperative serum carcinoembryonic antigen (CEA) levels; and body mass index (BMI). The TNM classification was defined according to the criteria of the American Joint Commission on Cancer (AJCC)/International Union Against Cancer (UICC) [16]. The tumor regression grade (TRG) was evaluated according to the AJCC system [17]. Perioperative outcomes were collected and evaluated, including surgical procedures, docking time, console time, operation time, estimated blood loss, time of the first flatus passage, time of resuming soft diet, duration of postoperative hospital stay, and postoperative first day visual analog scale (VAS) pain score.

Patients were regularly followed up, including the collection of their clinical outcomes and survival statuses. History-taking and physical examinations were performed postoperatively every 3 months during the first 2 years and then every 6 months during the following 3 years. Measure of serum CEA levels were performed every 2-3 months postoperatively. A colonoscopy was performed approximately 1 year after surgery. Repeat colonoscopy was typically recommended at 3 years, unless follow-up colonoscopy indicated advanced adenoma (villous polyp, polyp $>1 \mathrm{~cm}$, or high-grade dysplasia). Abdominal and pelvic CT scans were annually performed during postoperative 3 years in patients with stage II-III disease.

\section{Surgical procedure}

For all patients, we performed laparoscopic examinations to initially examine the intra-abdominal cavity. If an adhesion was observed, we performed laparoscopic lysis. Subsequently, we performed robotic surgery. The single-docking technique with five or six ports (Fig. 1) 


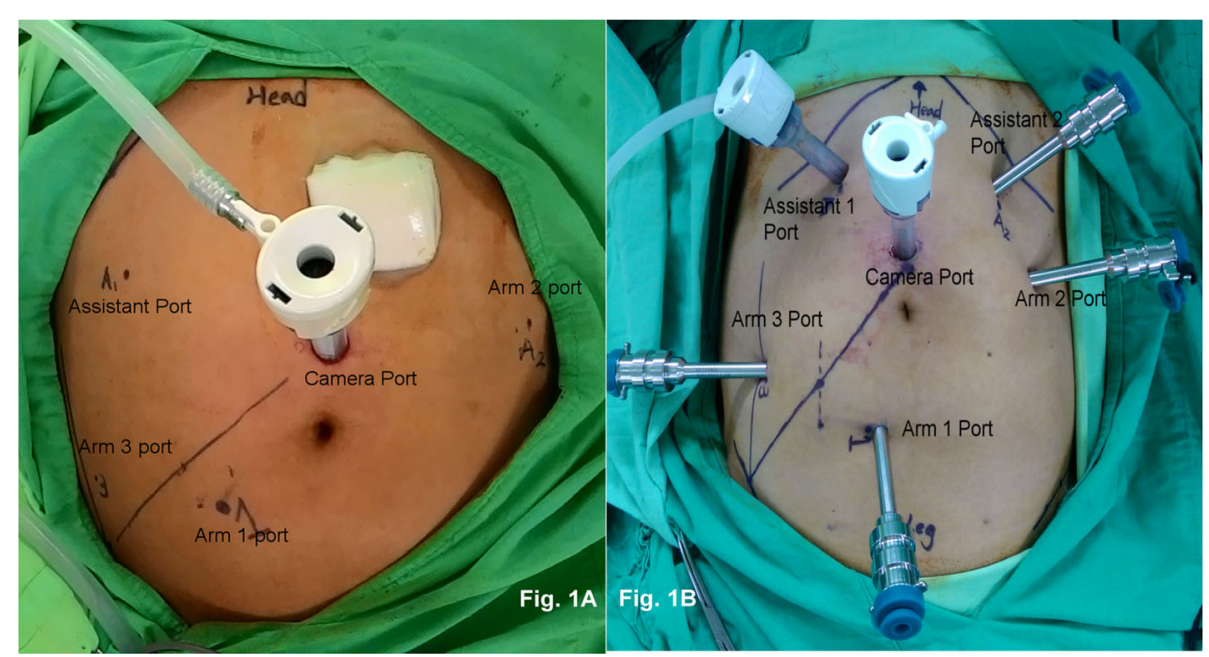

Fig. 1 a Port positions during single docking with the five-port technique. b Port positions during single docking with the six-port technique

was used as the docking method, as described in our previous studies $[18,19]$. One 12 -mm camera port was placed $2 \mathrm{~cm}$ superior to the umbilicus. One 8 -mm port (Arm 1 port) was inserted approximately $2 \mathrm{~cm}$ inferior to the line between the location of the camera port down to the right anterior superior iliac spine and slightly medial to the right mid-clavicular line (MCL). One $8-\mathrm{mm}$ port (Arm 3 port) was inserted right laterally $8 \mathrm{~cm}$ from the Arm 1 port. One 12-mm port (assistant port) was inserted at the right MCL, approximately $4 \mathrm{~cm}$ inferior to the right costal margin. One 8-mm port (Arm 2 port) was inserted left laterally $8 \mathrm{~cm}$ from the camera port. One 12-mm port (assistant port) was inserted at the left MCL, approximately $2-4 \mathrm{~cm}$ inferior to the left costal margin. A monopolar permanent cautery spatula (Intuitive Surgical) was used in Arm 1, a Maryland bipolar forceps (Intuitive Surgical) was used in Arm 2, and a double fenestrated grasper (Intuitive Surgical) was used in Arm 3. The da Vinci ${ }^{\circ}$ Si Surgical System was docked over the left flank of a patient. We performed medial to lateral dissection. Peritoneal incision at the level of the sacral promontory was performed first. The dissection was extended downward and then upward to the root of the IMA. We performed so-called high dissection and low ligation [19] in the form of D3 lymph node dissection and low-tie ligation of the IMA by using endo clips (Hem-O-Lok, Weck Closure Systems, NC) with preservation of the left colic artery in all patients. The inferior mesenteric vein (IMV) was also recognized, but was not ligated and divided instantly. If there was tension during the colonic anastomosis, the IMV would be ligated by using endo clips (Hem-O-Lok, Weck Closure Systems, NC) and divided. The splenic flexure of the colon was not routinely mobilized, if its mobilization was dependent on the tension of the anastomosis. Totally robotic-assisted TME with single-docking technique was performed in all patients.

After the sigmoid or descending colon, mesocolon, entire rectum and mesorectum were mobilized completely, low anterior resection (LAR) with the double-stapled technique, intersphincteric resection (ISR) with coloanal anastomosis and loop colostomy, or abdominoperineal resection (APR) was accordingly performed $[18,19]$., LAR with the doublestapled technique was used for a tumor located in the upper and mid rectum. The rectum was divided by the assistant using an Endo GIA stapler (Endo GIA ${ }^{\text {ti }}$ Reinforced Reload with Tri-Staple ${ }^{\mathrm{Tx}}$ Technology, Medtronic) or ECHELON FLEX $^{\text {sm }}$ Powered ENDOPATH ${ }^{\circ}$ stapler (Ethicon US, LLC) with one to three $60-\mathrm{mm}$ reloads before the da Vinci ${ }^{\circ} \mathrm{Si}$ Surgical System was undocked. We extracted the specimen through the extended camera port wound with the Alexis ${ }^{\circ}$ wound proctor and resected it. We then re-established the pneumoperitoneum and performed laparoscopic anastomosis by using a circular EEA stapler. Intraoperative dye test [20] was routinely performed to examine potential anastomotic leakage after LAR using the double-stapled technique. For a tumor located in the low rectum, ISR with coloanal anastomosis and loop colostomy was used. We used the Lone Star Retractor System ${ }^{\bullet}$ (Lone Star Medical Products Inc., Houston, TX) for ISR and subsequently we extracted the specimen and resected it transanally (natural orifice specimen extraction). Coloanal anastomosis was performed using the hand-sewn method. A protective loop colostomy of transverse colon was performed. Finally, we checked for bleeding in the abdominal cavity by using the traditional laparoscope and placed a drain tube in the pelvic cavity.

\section{Statistical analysis}

We used the Statistical Package for Social Sciences, Version 19.0 (SPSS Inc., Chicago, IL) to statistically analyze 
all data. All patients were followed up until their death, last follow-up, or December 31 2016. The time required to position the robot and secure the robotic arms to the corresponding port sites was defined as the docking time. The total time during which the surgeon performed any procedure by using the robotic system was defined as the console time. The time between the initial skin incision and wound closure completion was defined as the operation time. We analyzed the learning curves indicated by various console and operation times by using a seven-case simple moving average method. A $P$ value of $<0.05$ denoted statistical significance. Overall survival (OS) was defined as the time from the date of primary treatment to the date of death from any cause or the date of last follow-up. Disease-free survival (DFS) was defined as the time from the date of primary treatment to the date of diagnosis of recurrence or metastatic disease or the date of last follow-up. OS and DFS were calculated by using the Kaplan-Meier method.

\section{Results}

\section{Patients' characteristics and perioperative outcomes}

The baseline characteristics and perioperative outcomes of 95 patients with rectal cancer who underwent totally robotic-assisted TME with the single-docking technique were summarized in Table 1 . The median age and BMI of the patients was 62 (range, 28-88) years and 23.54 (range, $17.20-34.02) \mathrm{kg} / \mathrm{m}^{2}$, respectively. Of the 95 patients, 48 (50.5\%), 30 (31.6\%), 17 (17.9\%) had lower, middle, and upper rectal cancers, respectively. The median distance of the tumor from the anal verge was 5.5 (range, 1.0-15.0) $\mathrm{cm}$.

The most frequent surgical procedure was LAR (59/ 95, 62.1\%). ISR with coloanal anastomosis was performed in $32(33.7 \%)$ patients, and APR was performed in $4(4.2 \%)$ patients. Moreover, of the 32 patients undergoing ISR, 3 underwent transabdominal ISR and their tumor distances from the anal verge were $2-4 \mathrm{~cm}$. Positive dye leakage after the completion of anastomosis was identified in six patients who had undergone LAR. Protective colostomies were performed accordingly. Finally, protective diverting loop transverse colostomy was performed in 38 (43.9\%) patients, including 32 patients and 6 patients who underwent ISR and LAR, respectively. Sphincter preservation rate was $95.8 \%$. The median estimated blood loss including tissue fluid after CCRT was $80 \mathrm{~mL}$. The median time of the first flatus passage and resuming soft diet postoperatively was 2 and 4 days, respectively. The median duration of postoperative hospital stay was 6 days (range, 5-32).

\section{Postoperative complications}

The postoperative complications are summarized in Table 2. Postoperative complications were observed in
14 patients with 17 episodes (17.9\%). Three patients who developed intraabdominal abscess, CT-guided pigtail drainage were subsequently performed in 2 patients. Anastomosis leakage was observed in 5 (5.4\%) patients who underwent LAR with the double-stapled technique, and loop colostomy of transverse colon was subsequently performed. Four (4.2\%) patients developed stenosis of coloanal anastomosis and underwent dilation using a colonoscope. Urethral injury during ISR was noted in one $(1.0 \%)$ patients. According to the ClavienDindo Classification, all post-operative ileus, urinary tract, and pulmonary complications were of grades I, and the patients recovered after conservative treatment. Moreover, no 30-day hospital mortality occurred.

\section{Pathological outcomes and oncological outcomes}

The pathological characteristics and oncological outcomes of all 95 patients are listed in Table 3. Preoperative clinical staging demonstrated that the majority of the patients had locally advanced rectal cancers including T3 in 61 (64.2\%) patients, T4 in 13 (13.7\%) patients, or N+ in 57 (60.0\%) patients. Therefore, preoperative CCRT was performed in 75 (78.9\%) patients, including FOLFOX regimen in 58 (77.3\%) patients with cT4 or $\mathrm{cN}+$ disease, fluoropyrimidine-based regimen in 17 (22.7\%) patients. The median number of harvested lymph nodes and apical lymph nodes was 9 (range, 0-36) and 2 (range, 0-15), respectively. However, positive apical lymph node metastasis was observed in only 3 (2.9\%) patients. The median distance of the distal resection margin (DRM) and circumferential resection margin (CRM) was 2.30 and $1.0 \mathrm{~cm}$, respectively. CRM and DRM were positive in $2(2.1 \%)$ and 1 (1.1\%) patients, respectively. R0 resection for primary rectal cancer was performed in 92 (96.8\%) patients. Of the 75 patients who received preoperative CCRT, a pathologic complete response (pCR) of the primary tumor was observed in $27(28.4 \%)$ patients. 28 (37.3\%), 30 (40.0\%), 11 (14.7\%), and $6(8.0 \%)$ patients exhibited complete response (TRG 0), moderate response (TRG 1), minimal response (TRG 2), and poor response (TRG 3), respectively. The median time interval between radiotherapy completion and robotic surgery was 82 (range, 41-203) days.

The median follow-up duration of 95 patients from the primary treatment was 25.6 (range, 6.6-52.2) months. One patient undergoing local excision of primary tumor and radiotherapy at other hospital underwent chemotherapy with FOLFOX regiment and robotic ISR and coloanal anastomosis at our hospital after local recurrent tumor developed. We excluded this patient to analyze the oncological outcomes of the patients with undergoing R0 resection. Of 91 patients undergoing R0 resection, local recurrence and distant metastases were noted in 5 (5.5\%) and $10(11.0 \%)$ patients, respectively. At a median followup duration of 25.6 months, the 2-year OS was $94 \%$ and 2-year DFS was $83 \%$ (Fig. 2). Furthermore, 2-year local 
Table 1 Baseline characteristics and perioperative outcomes of 95 patients with stage 0 -III rectal cancer undergoing robotic-assisted total mesorectal excision

\begin{tabular}{|c|c|}
\hline Characteristic & \\
\hline Age (years, median) (range) & $62(28-88)$ \\
\hline \multicolumn{2}{|l|}{ Gender } \\
\hline Female & $35(36.8 \%)$ \\
\hline Male & $60(63.2 \%)$ \\
\hline \multicolumn{2}{|l|}{ Tumor distance from anal verge $(\mathrm{cm})$} \\
\hline$\leqq 5$ (Lower) & $48(50.5 \%)$ \\
\hline 6-10 (Middle) & $30(31.6 \%)$ \\
\hline 11-15 (Upper) & $17(17.9 \%)$ \\
\hline Distance from anal verge (cm, median) (range) & $5.5(1-15)$ \\
\hline \multicolumn{2}{|l|}{ Pre-operation CCRT } \\
\hline Yes & 75 (78.9\%) \\
\hline No & $20(21.1 \%)$ \\
\hline Pre-operation chemotherapy regimen & 75 \\
\hline FOLFOX & $58(77.3 \%)$ \\
\hline Fluoropyrimidine-based & $17(22.7 \%)$ \\
\hline $\begin{array}{l}\text { Time interval between radiotherapy completion and robotic surgery (day, median) } \\
\text { (range) ( } 75 \text { patients undergoing pre-operation chemotherapy) }\end{array}$ & $82(41-203)$ \\
\hline \multicolumn{2}{|l|}{ ASA classification } \\
\hline$\|$ & $52(54.7 \%)$ \\
\hline III & $43(45.3 \%)$ \\
\hline BMI kg/m² (Median) (range) & $23.54(17.20-34.02)$ \\
\hline \multicolumn{2}{|l|}{ Procedure } \\
\hline LAR & $59(62.1 \%)$ \\
\hline ISR & $\begin{array}{l}32(33.7 \%) \text { (including } 3 \\
\text { transabdominal ISR) }\end{array}$ \\
\hline APR & $4(4.2 \%)$ \\
\hline \multicolumn{2}{|l|}{ Protective Diverting Colostomy } \\
\hline Yes & $38(40.0 \%)$ \\
\hline No & $57(60.0 \%)$ \\
\hline Docking Time (min, median) (range) & $5(3-22)$ \\
\hline Console Time (min, median) (range) & $200(130-435)$ \\
\hline Operation Time (min, median) (range) & $325(210-795)$ \\
\hline Estimated blood loss (mL, Median) & $80(15-1050)$ \\
\hline Time of first flatus passage (day) (Median, range) & $2(1-10)$ \\
\hline Time of resuming soft diet (day) (Median, range) & $4(2-15)$ \\
\hline Postoperative hospital stay (day) (Median, range) & $6(5-32)$ \\
\hline Postoperative first day VAS pain score (Median, range) & $3(1-8)$ \\
\hline
\end{tabular}

APR abdominoperineal resection, AR anterior resection, ASA American Society of Anesthesiologists, BMI Body mass index, CCRT Concurrent chemoradiotherapy, ISR, intersphenteric resection, LAR low anterior resection, VAS visual analog scale

control rate and 2-year distant metastasis control rate were $95 \%$ and $90 \%$, respectively.

\section{Learning curve of robotic CRC surgery}

The learning curves in terms of console and operation time are presented in Fig. 3. The median docking, console and operation time was 5 (range, 3-22), 200 (range, 130-435), and 325 (range, 210-795) minutes, respectively. A linear regression analysis indicated a decreasing trend for console time. The first plateau of console time was observed after 32 patients. The mean console time for the first 32 patient was significantly 
Table 2 Postoperative complications in 95 patients with stage 0-III rectal cancer undergoing robotic-assisted total mesorectal excision

\begin{tabular}{lll}
\hline Complications & Number (\%) & Management \\
\hline Post-operative bleeding & $1(1.0 \%)$ & Laparotomy \\
Intra-abdominal infection/abscess & $3(3.2 \%)$ & 2: conservative treatment \\
& & $1:$ CT-guided pig-tail drainage \\
Coloanal Anastomosis Stenosis & $4(4.2 \%)$ & Colonoscopic dilation \\
lleus & $1(1.0 \%)$ & Conservative treatment \\
Anastomosis leakage & $5(5.4 \%)$ & Loop transverse colostomy \\
Urethral injury & $1(1.0 \%)$ & Conservative treatment \\
Pulmonary complication & $2(2.1 \%)$ & Conservative treatment \\
Total & $17(17.9 \%)$ &
\end{tabular}

longer than that of the remaining patients $(270.09 \pm$ 64.830 vs $200.27 \pm 42.080 \mathrm{~min}, P<0.001)$. The linear regression analysis of operation time also indicated a decreasing trend for operation time. The mean operation time for the first 32 patient was significantly longer than that of the remaining patients $(516.09 \pm 11.460$ vs 306.03 $\pm 6.804 \mathrm{~min}, P<0.001)$.

\section{Discussion}

In this study, we presented our experiences and shortterm clinical and oncological outcomes of 95 patients with stage I-III rectal cancer who underwent totally robotic-assisted TME with the single-docking technique. The single-docking technique was performed in the complete procedure of totally robotic-assisted radical rectal cancer surgery without moving the robotic surgical cart and repositioning robotic arms. Meanwhile, we demonstrate that this technique is safe and feasible for patients with rectal cancer, with or without preoperative CCRT. Upmost important, favorable short-term clinical and oncological outcomes can be achieved by combining this approach with appropriate preoperative CCRT.

The hybrid technique was the first technique used in robotic rectal surgery, and many robotic rectal surgeries have been performed using the hybrid technique. However, with the hybrid technique, the advantages of the robotic system could not be utilized during the laparoscopic phase. The dual docking technique requires the movement of the robotic surgical cart and repositioning of robotic arms [21]. Hellan et al. first performed a robotic rectal surgery by using the hybrid technique [22] and then by using the single-docking technique [23]. Ahmed et al. [15] reported the experience and clinical outcomes of 100 patients who underwent robotic rectal surgery with the single-docking modified flip-arm technique. Luca et al. [24] used the single-docking technique to perform mobilization of the splenic flexure and TME. The surgical cart was not moved and the robotic arms were not repositioned during the surgery. The port sites of robotic arms used in this present study were different from those used in the study of Luca et al. [24].

In our study, the mean console time of the first 32 patients was significantly longer than that of the remaining patients. By using a standardized approach and more practice, robotic rectal surgery with TME can be performed safely and the console time can be reduced significantly. The results of this study were consistent with those of a meta-analysis conducted by Scarpinata et al. [25]. The selection criteria for robotic surgery in this meta-analysis were obesity, male sex, preoperative radiotherapy, and tumors in the lower two-thirds of the rectum. Though 78 (82.1\%) patients had middle to low rectal cancers, the pCR was in $28.4 \%$ of patients and TRG 0 and 1 in $77.3 \%$ of patients. The pCR rate observed in our study (28.6\%) is relatively higher than that reported in previous studies (10-30\%, with less than $20 \%$ in most of studies) $[26,27]$. The sphincter preservation rate achieved in our study was $96.1 \%$, which is comparable with that reported by Kim et al. [28] and Saklani et al. [29] (Table 4).

TME completeness is a representative of the quality of rectal cancer surgery. The two crucial parameters of TME completeness are CRM involvement and DRM distance. Moreover, CRM involvement has been reported as a prognostic factor for local recurrence and survival [30-33]. In this study, the rate of CRM involvement was $2.1 \%$, with a median distance of $1.0 \mathrm{~cm}$, which is comparable with that reported in the previous studies (0-16.1\%) (Table 4). Moreover, the rate of DRM involvement was $1.1 \%$ with a median distance of $2.3 \mathrm{~cm}$, which is comparable to that reported in the previous studies $(1.5-3.9 \mathrm{~cm})$ (Table 4$)$. R0 resection for primary rectal cancer was performed in 92 (96.8\%) patients. Of the 91 patients with undergoing R0 resection, 5 (5.5\%) developed local recurrence and 10 (11.0\%) developed distant metastasis.

Although $82.1 \%$ of our patients had middle to low rectal cancers with a median distance of $5.5 \mathrm{~cm}$ from the anal verge and $63.2 \%$ of our patients were men, we did not mobilize the splenic flexure in most of our patients and still could perform precise dissection during TME 
Table 3 Clinicopathologic characteristics and oncological outcomes of 95 patients with stage 0-III rectal cancer undergoing robotic-assisted total mesorectal excision

\begin{tabular}{|c|}
\hline Preoperative clinical staging \\
\hline Tumor depth \\
\hline $\mathrm{T} 1$ \\
\hline $\mathrm{T} 2$ \\
\hline T3 \\
\hline T4 \\
\hline Lymph Node metastasis \\
\hline No \\
\hline N1 \\
\hline N2 \\
\hline AJCC ${ }^{a}$ Stage (Clinical) \\
\hline । \\
\hline$\|$ \\
\hline III \\
\hline Postoperative pathological outcomes \\
\hline Histology \\
\hline Well differentiation \\
\hline Moderate differentiation \\
\hline Poor differentiation \\
\hline Tumor size \\
\hline$<5 \mathrm{~cm}$ \\
\hline$\geq 5 \mathrm{~cm}$ \\
\hline
\end{tabular}

Tumor size ( $\mathrm{cm}$, mean $\pm \mathrm{SD})$ (range)

Tumor depth

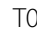

Tis

$\mathrm{T} 1$

$\mathrm{T} 2$

T3

T4

Lymph Node metastasis

$$
\text { NO }
$$$$
\text { N1 }
$$$$
\text { N2 }
$$

AJCC Stage (Pathologic)

$$
0
$$$$
\text { । }
$$$$
\text { ॥ }
$$$$
\text { III }
$$

Tumor Regression Grade (75 patients with preoperative CCRT)

$$
1
$$$$
0
$$

$16(16.9 \%)$

$76(80.0 \%)$

$3(3.1 \%)$

85 (89.5\%)

10 (10.5\%)

$29(30.5 \%)$

$1(1.0 \%)$

$14(14.7 \%)$

$20(21.1 \%)$

28 (29.5\%)

3 (3.2\%)

73 (77.1\%)

19 (19.8\%)

3 (3.1\%)

27 (28.4\%)

$27(28.4 \%)$

$19(20.0 \%)$

$22(23.2 \%)$

$28(37.3 \%)$

$30(40.0 \%)$

\begin{tabular}{|c|c|}
\hline 3 & $6(8.0 \%)$ \\
\hline Harvested Lymph Node (median) (range) & $9(0-36)$ \\
\hline Harvested Apical Node (median) (range) & $2(0-15)$ \\
\hline $\begin{array}{l}\text { Distance of distal resection margin }(\mathrm{cm}, \\
\text { median) (range) }\end{array}$ & $2.30(0.2-6.5)$ \\
\hline $\begin{array}{l}\text { Distance of circumferential resection margin } \\
(\mathrm{cm} \text {, median) (range) }\end{array}$ & $1.0(0.2-3.5)$ \\
\hline \multicolumn{2}{|l|}{ Distal resection margin } \\
\hline Free & 94 (98.9\%) \\
\hline Positive & $1(1.1 \%)$ \\
\hline \multicolumn{2}{|l|}{ Circumferential resection margin } \\
\hline Free & $93(97.9 \%)$ \\
\hline Positive & $2(2.1 \%)$ \\
\hline \multicolumn{2}{|l|}{ Resection Degree of Primary tumor } \\
\hline Ro & $92(96.8 \%)$ \\
\hline R1 & $3(3.2 \%)$ \\
\hline \multicolumn{2}{|l|}{ Oncological outcomes } \\
\hline Follow-up periods (months, median) (range) & $25.6(6.6-52.2)$ \\
\hline RO resection & 91 \\
\hline Locoregional recurrence & $5(5.5 \%)$ \\
\hline Distant metastasis & $10(11.0 \%)$ \\
\hline Liver + Lung & $1(1.1 \%)$ \\
\hline Lung & $5(5.5 \%)$ \\
\hline Liver & $2(2.2 \%)$ \\
\hline Chest Wall & $1(1.1 \%)$ \\
\hline Peritoneal carcinomatosis & $1(1.1 \%)$ \\
\hline R1 resection & 3 \\
\hline Local recurrence & $1(33.3 \%)$ \\
\hline Lung & $1(33.3 \%)$ \\
\hline Peritoneum & 1 (33.3\%) \\
\hline
\end{tabular}

$11(14.7 \%)$
Table 3 Clinicopathologic characteristics and oncological outcomes of 95 patients with stage 0 -III rectal cancer undergoing robotic-assisted total mesorectal excision (Continued)

$2.46 \pm 1.652(0-8)$

AJCC American Joint Commission on Cancer

procedure even using our single-docking technique. However, we still achieved a comparable distance of DMR and favorable negative rates of DRM and CRM. Protective diverting colostomy was performed in $40.0 \%$ of the patients undergoing sphincter preservation surgery; however, the anastomosis leakage rate in our study was comparable with that reported in the literature (Table 4).

The single-docking technique used in the present study is safe and feasible for treating patients with rectal cancer. However, some technical problems still exist. External collisions of the robotic arms usually occur. By using a standardized approach and through more practice, the positions of the robotic arms can be determined and external collisions can be avoided. We always encountered arm collisions when performing pelvic 

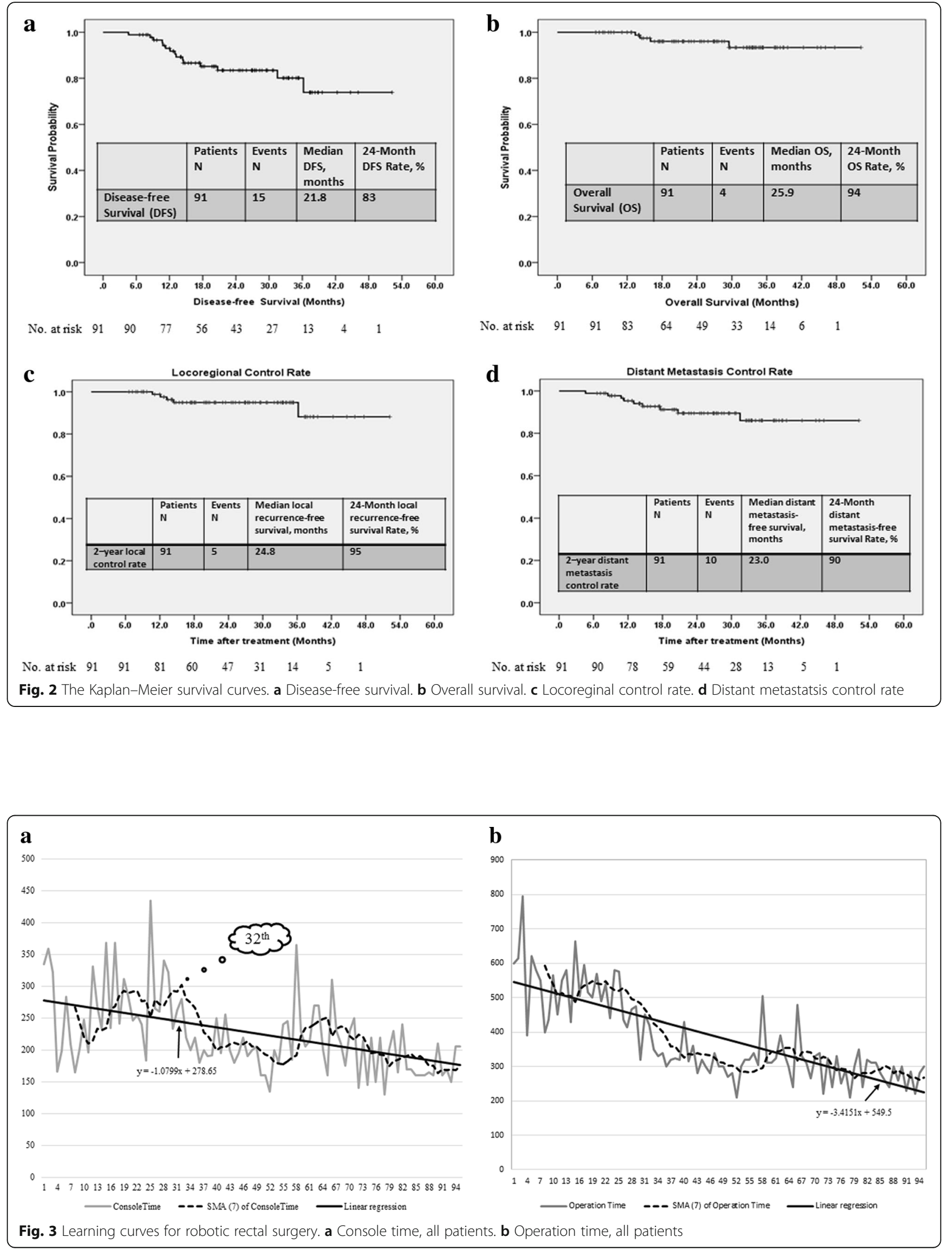


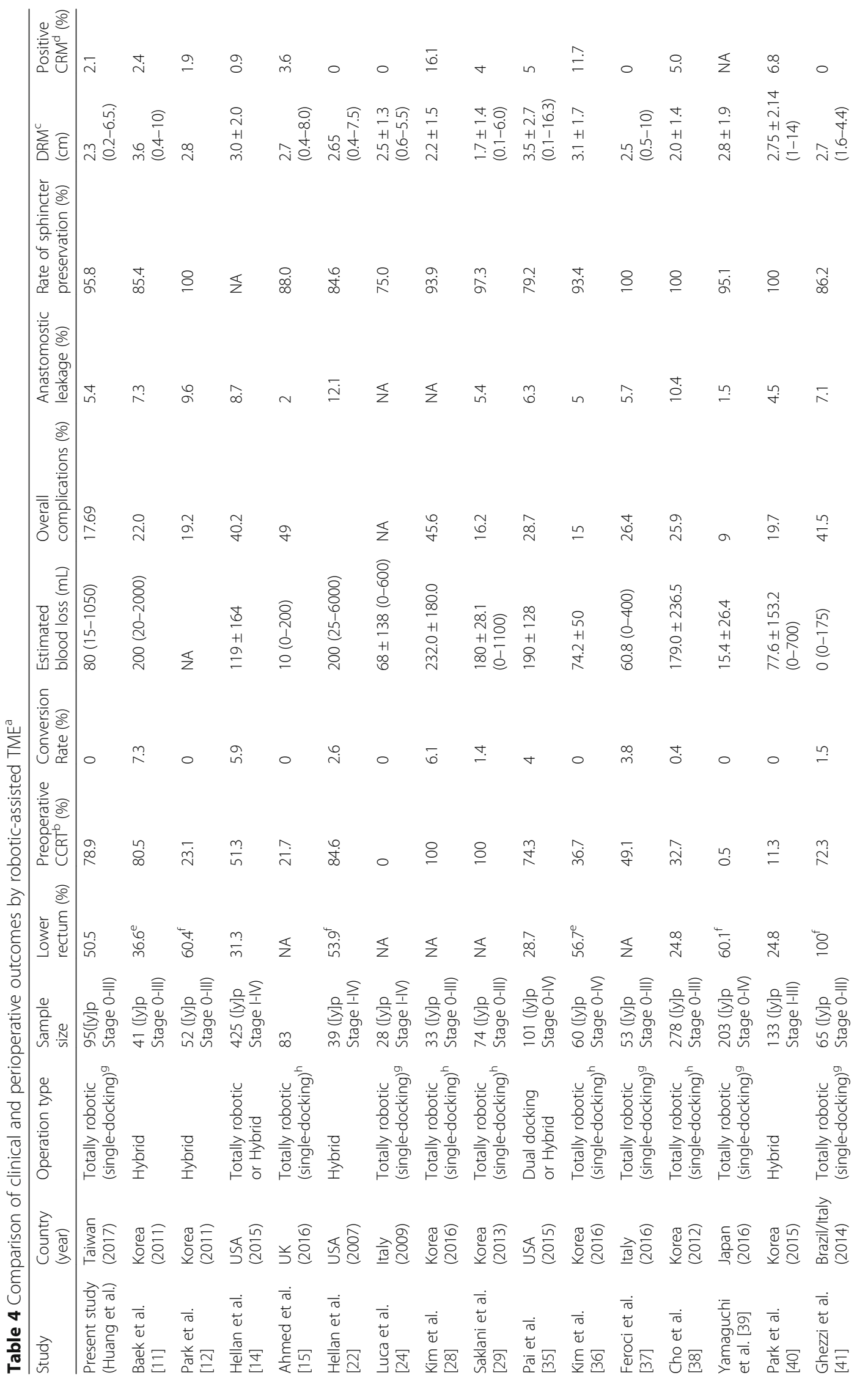




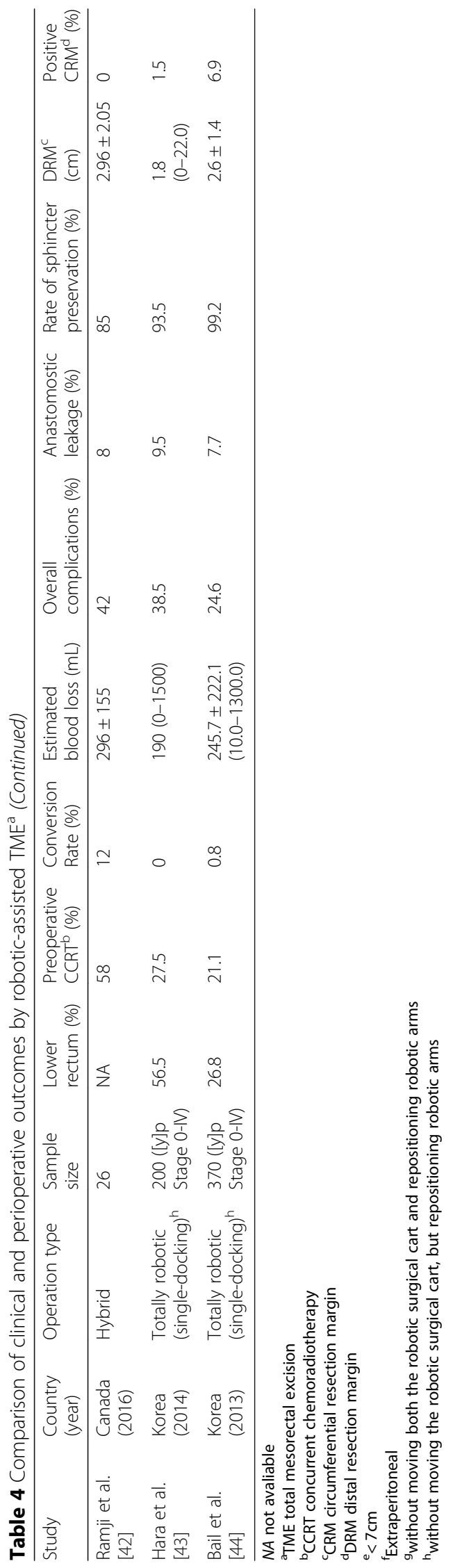


Table 5 Comparison of short-term oncological outcomes by robotic-assisted TME ${ }^{a}$

\begin{tabular}{llllll}
\hline Study & Country (year) & Local recurrence (\%) & Distant metastasis (\%) & Disease-free survival & Overall survival \\
\hline Present study (Huang et al.) & Taiwan (2017) & 2.4 & 14.5 & $83.0 \%(2$-year) & $95.0 \%(2$-year) \\
Pai et al. [35] & USA (2015) & 4 & 17 & $79.2 \%(3$-year) & $90.1 \%(3-$ year) \\
Kim et al. [36] & Korea (2016) & 1.9 & 26.4 & $72.8 \%(4-$ year) & $87.7 \%(4-$ year) \\
Feroci et al. [37] & Italy (2016) & 1.9 & 17 & $79.2 \%(3$-year) & $90.2 \%(3-$ year) \\
Cho et al. [38] & Korea (2012) & 1.8 & 12.2 & $81.8 \%(5$-year) & $92.2 \%(5$-year) \\
Park et al. [40] & Korea (2015) & 2.3 & 12.0 & $81.9 \%(5$-year) & $92.8 \%(5$-year) \\
Ghezzi et al. [41] & Brazil/Italy (2014) & 3.2 & 18.5 & $73.2 \%(5$-year) & $85.2 \%(5$-year) \\
Hara et al. [43] & Korea (2014) & 4.5 & 10 & $81.7 \%(5$-year) & $92.0 \%(5$-year) \\
Bail et al. [44] & Korea (2013) & 3.6 & 17.6 & $79.2 \%(3$-year) & $93.1 \%(3-$ year) \\
\hline
\end{tabular}

${ }^{\mathrm{a}} T M E$ total mesorectal excision

dissection. To reduce the occurrence of arm collisions, we used a monopolar permanent cautery spatula in Arm 3 and a double fenestrated grasper in Arm 1. Complete mobilization of the splenic flexure through our singledocking technique is difficult. When it was necessary to mobilize the splenic flexure, we reset the setting of the robotic arms (flip-arm techniques) to enable the surgeon to control different robotic arms rather than redocking the surgical cart. The single-docking technique with six ports (two assistant ports) is recommended for situations where performing pelvic dissection is difficult, such as for patients with mid and low rectal cancers, a high BMI, narrow pelvis, heavy mesorectum, or T4 lesions; women with huge uterine myomas; and patients who have responded poorly to neoadjuvant CCRT.

This study has some limitations that should be addressed. First, this is a single-institution retrospective study including only 95 patients. Second, the interval of follow-up was short, with 25.6 months of median followup duration; thus, only short-term (2 year) survival and oncological outcomes were reported. Nevertheless, 2-year OS (94\%) and the 2-year DFS (83\%) observed in our study were consistent with those reported in previous studies (Table 5). Furthermore, 2-year local control rate (95\%) and 2-year distant metastasis control rate $(90 \%)$ were consistent with those reported in previous studies (Table 5) [34]. Third, we did not evaluate the postoperative outcomes of urinary and sexual functions.

\section{Conclusions}

With comparable short-term clinical outcomes, we demonstrate that this technique is safe and feasible for patients with rectal cancer, with or without preoperative CCRT. Moreover, favorable short-term oncological outcomes can be achieved by combining this approach with appropriate preoperative CCRT. However, long-term oncological outcomes should be further investigated by conducting studies having a longer follow-up duration.

\section{Abbreviations}

AJCC: American Joint Commission on Cancer; APR: Abdominoperineal resection; BMI: Body mass index; CCRT: Concurrent chemoradiotherapy; CEA: Carcinoembryonic antigen; CRC: Colorectal cancer; CRM: Circumferential resection margin; CT: Computed tomography; DFS: Disease-free survival; DRM: Distal resection margin; IMA: Inferior mesentery artery; IMV: Inferior mesentery vein; ISR: Intersphincteric resection; LAR: Low anterior resection; LARC: Locally advanced rectal cancer; LCA: Left colic artery; LCRT: Longcourse radiotherapy; MRI: Magnetic resonance imaging; OS: Overall survival; pCR: Pathologic complete response; SMA: Simple moving average; TME: Total mesorectal excision; TRG: Tumor regression grade; UICC: International Union Against Cancer; VAS: Visual analog scale

\section{Acknowledgements}

None

\section{Funding}

This work was supported by grants from the Excellence for Cancer Research Center through funding by the Ministry of Science and Technology (MOST105-2325-B-037-001) and the Ministry of Health and Welfare (MOHW106-TDU-B-212-144,007); Health and Welfare Surcharge of Tobacco Products, Taiwan, Republic of China; and Kaohsiung Medical University Hospital (KMUH98-8G06, KMHU100-0 M14, KMUHS10522, KMUHS10505, KMUHS10418, and KMUHGCRC2016002). The study was supported by the Kaohsiung Medical University "Aim for the top University Grant" (KMUTP105C01, KMU-TP105C11, KMU-TP106005, KMU-TP105A14, KMU-

DK106005, KMU-S105011, and SH000113 [Give2Asia]) and the Grant from Biosignature in Colorectal Cancers, Academia Sinica, Taiwan.

\section{Availability of data and materials}

The datasets used and/or analysed during the current study are available from the corresponding author on reasonable request.

\section{Authors' contributions}

CWH analyzed the data and wrote the manuscript. HLT, YSY, WCS, MYH, $\mathrm{CMH}$, and YTC made substantial contributions in data acquisition, statistical analyses, and data interpretation, and helped in manuscript preparation. JYW participated in study design and coordination. All authors have read and approved the final manuscript.

Ethics approval and consent to participate

This study was approved by the institutional review board of the Kaohsiung Medical University Hospital (KMUHIRB-E-20150003). Written informed consent to participate was obtained from each patient before performing the robotic surgery.

\section{Consent for publication}

Written informed consent for publication was obtained from the patients for publication of this case report and any accompanying images. A copy of the written consent is available for review by the Editor of this journal. 


\section{Competing interests}

The authors declare that they have no competing interests.

\section{Publisher's Note}

Springer Nature remains neutral with regard to jurisdictional claims in published maps and institutional affiliations.

\section{Author details}

'Graduate Institute of Medicine, College of Medicine, Kaohsiung Medical University, Kaohsiung, Taiwan. ${ }^{2}$ Division of Colorectal Surgery, Department of Surgery, Kaohsiung Medical University Hospital, Kaohsiung Medical University, Kaohsiung, Taiwan. ${ }^{3}$ Division of Trauma, Department of Surgery, Kaohsiung Medical University Hospital, Kaohsiung Medical University, Kaohsiung, Taiwan. ${ }^{4}$ Division of General Surgery Medicine, Department of Surgery, Kaohsiung Medical University Hospital, Kaohsiung Medical University, Kaohsiung, Taiwan. ${ }^{5}$ Department of Surgery, Faculty of Medicine, College of Medicine, Kaohsiung Medical University, Kaohsiung, Taiwan. ${ }^{6}$ Graduate Institute of Clinical Medicine, College of Medicine, Kaohsiung Medical University, Kaohsiung, Taiwan. Department of Radiation Oncology, Kaohsiung Medical University Hospital, Kaohsiung Medical University, Kaohsiung, Taiwan. ${ }^{8}$ Division of Pediatric Surgery, Department of Surgery, Kaohsiung Medical University Hospital, Kaohsiung Medical University, Kaohsiung, Taiwan. ${ }^{9}$ Center for Biomarkers and Biotech Drugs, Kaohsiung Medical University, Kaohsiung, Taiwan. ${ }^{10}$ Center for Environmental Medicine, Kaohsiung Medical University, Kaohsiung, Taiwan. ${ }^{11}$ Research Center for Natural products \& Drug Development, Kaohsiung Medical University, Kaohsiung, Taiwan.

Received: 1 August 2017 Accepted: 20 November 2017 Published online: 05 December 2017

\section{References}

1. Heald RJ, Husband EM, Ryall RD. The mesorectum in rectal cancer surgery — the clue to pelvic recurrence? Br J Surg. 1982;69:613-6.

2. MacFarlane JK, Ryall RD, Heald RJ. Mesorectal excision for rectal cancer. Lancet. 1993:341:457-60.

3. Sauer R, Liersch T, Merkel S, Fietkau R, Hohenberger W, Hess C, et al. Preoperative versus postoperative chemoradiotherapy for locally advanced rectal cancer: results of the German CAO/ARO/AIO-94 randomized phase III trial after a median follow-up of 11 years. J Clin Oncol. 2012;30:1926-33.

4. Sauer R, Becker H, Hohenberger W, Rödel C, Wittekind C, Fietkau R, et al. Preoperative versus postoperative chemoradiotherapy for rectal cancer. $\mathrm{N}$ Engl J Med. 2004;351:1731-40.

5. McCarthy K, Pearson K, Fulton R, Hewitt J. Pre-operative chemoradiation for non-metastatic locally advanced rectal cancer. Cochrane Database Syst Rev. 2012;12:CD008368

6. Bosset JF, Calais G, Mineur L, Maingon P, Radosevic-Jelic L, Daban A, et al. Enhanced tumorocidal effect of chemotherapy with preoperative radiotherapy for rectal cancer: preliminary results-EORTC 22921. J Clin Oncol. 2005;23:5620-7.

7. Gérard JP, Conroy T, Bonnetain F, Bouché O, Chapet O, Closon-Dejardin $M T$, et al. Preoperative radiotherapy with or without concurrent fluorouracil and leucovorin in T3-4 rectal cancers: results of FFCD 9203. J Clin Oncol. 2006:24:4620-5.

8. van der Pas MH, Haglind E, Cuesta MA, Fürst A, Lacy AM, Hop WC, et al. Laparoscopic versus open surgery for rectal cancer (COLOR II): short-term outcomes of a randomised, phase 3 trial. Lancet Oncol. 2013;14:210-8.

9. Guillou PJ, Quirke P, Thorpe H, Walker J, Jayne DG, Smith AM, et al. Shortterm endpoints of conventional versus laparoscopic-assisted surgery in patients with colorectal cancer (MRC CLASICC trial): multicentre, randomised controlled trial. Lancet. 2005;365:1718-26.

10. Weber PA, Merola S, Wasielewski A, Ballantyne GH. Telerobotic-assisted laparoscopic right and sigmoid colectomies for benign disease. Dis Colon Rectum. 2002;45:1689-94.

11. Baek JH, Pastor C, Pigazzi A. Robotic and laparoscopic total mesorectal excision for rectal cancer: a case-matched study. Surg Endosc. 2010;25: 521-5.

12. Park JS, Choi GS, Lim KH, Jang YS, Jun SH. S052: a comparison of robotassisted, laparoscopic, and open surgery in the treatment of rectal cancer. Surg Endosc. 2011;25:240-8.
13. Kim JY, Kim NK, Lee KY, Hur H, Min BS, Kim JH. A comparative study of voiding and sexual function after total mesorectal excision with autonomic nerve preservation for rectal cancer: laparoscopic versus robotic surgery. Ann Surg Oncol. 2012:19:2485-93.

14. Hellan M, Ouellette J, Lagares-Garcia JA, Rauh SM, Kennedy HL, Nicholson JD, et al. Robotic rectal cancer resection: a retrospective multicenter analysis. Ann Surg Oncol. 2015;22:2151-8.

15. Ahmed J, Nasir M, Flashman K, Khan J, Parvaiz A. Totally robotic rectal resection: an experience of the first 100 consecutive cases. Int J Color Dis. 2016;31:869-76.

16. Edge SB, Byrd DR, Compton CC, et al. AJCC cancer staging manual. 7th ed. New York: Springer; 2010.

17. Mace AG, Pai RK, Stocchi L, Kalady MF. American Joint Committee on Cancer and College of American Pathologists regression grade: a new prognostic factor in rectal cancer. Dis Colon Rectum. 2015:58:32-44.

18. Huang CW, Yeh YS, Ma CJ, Choy TK, Huang MY, Huang CM, et al. Robotic colorectal surgery for laparoscopic surgeons with limited experience: preliminary experiences for 40 consecutive cases at a single medical center. BMC Surg. 2015;15:73.

19. Huang CW, Yeh YS, Su WC, Tsai HL, Choy TK, Huang MY, et al. Robotic surgery with high dissection and low ligation technique for consecutive patients with rectal cancer following preoperative concurrent chemoradiotherapy. Int J Color Dis. 2016;31:1169-77.

20. Chen CW, Chen MJ, Yeh YS, Tsai HL, Chang YT, Wang JY. Intraoperative anastomotic dye test significantly decreases incidence of anastomotic leaks in patients undergoing resection for rectal cancer. Tech Coloproctol. 2013;17:579-83.

21. Sun Y, Xu H, Li Z, Han J, Song W, Wang J, et al. Robotic versus laparoscopic low anterior resection for rectal cancer: a meta-analysis. World J Surg Oncol. 2016;14:61.

22. Hellan M, Anderson C, Ellenhorn JD, Paz B, Pigazzi A. Short-term outcomes after robotic-assisted total mesorectal excision for rectal cancer. Ann Surg Oncol. 2007;14:3168-73.

23. Hellan $M$, Stein $H$, Pigazzi A. Totally robotic low anterior resection with total mesorectal excision and splenic flexure mobilization. Surg Endosc. 2009;23:447-51.

24. Luca F, Cenciarelli S, Valvo M, Pozzi S, Faso FL, Ravizza D, et al. Full robotic left colon and rectal cancer resection: technique and early outcome. Ann Surg Oncol. 2009;16:1274-8.

25. Scarpinata $\mathrm{R}$, Aly EH. Does robotic rectal cancer surgery offer improved early postoperative outcomes? Dis Colon Rectum. 2013;56:253-62.

26. Madbouly KM, Hussein AM. Changing operative strategy from abdominoperineal resection to sphincter preservation in T3 low rectal cancer after downstaging by neoadjuvant chemoradiation: a preliminary report. World J Surg. 2015;39:1248-56.

27. Maas M, Nelemans PJ, Valentini $V$, Das P, Rödel C, Kuo LJ, et al. Long-term outcome in patients with a pathological complete response after chemoradiation for rectal cancer: a pooled analysis of individual patient data. Lancet Oncol. 2010;11:835-44.

28. Kim YS, Kim MJ, Park SC, Sohn DK, Kim DY, Chang HJ, et al. Robotic versus laparoscopic surgery for rectal cancer after preoperative chemoradiotherapy: case-matched study of short-term outcomes. Cancer Res Treat. 2016:48:225-31.

29. Saklani AP, Lim DR, Hur H, Min BS, Baik SH, Lee KY, et al. Robotic versus laparoscopic surgery for mid-low rectal cancer after neoadjuvant chemoradiation therapy: comparison of oncologic outcomes. Int J Color Dis. 2013;28:1689-98.

30. Adam IJ, Mohamdee MO, Martin IG, Scott N, Finan PJ, Johnston D, et al. Role of circumferential margin involvement in the local recurrence of rectal cancer. Lancet. 1994;344:707-11.

31. Quirke P, Steele R, Monson J, Grieve R, Khanna S, Couture J, et al. Effect of the plane of surgery achieved on local recurrence in patients with operable rectal cancer: a prospective study using data from the MRC CR07 and $\mathrm{NCIC}$ CTG CO16 randomised clinical trial. Lancet. 2009;373:821-8.

32. Quirke $\mathbf{P}$. Training and quality assurance for rectal cancer: 20 years of data is enough. Lancet Oncol. 2003;4:695-702.

33. Kwak JM, Kim SH. Robotic surgery for rectal cancer: an update in 2015 Cancer Res Treat. 2016:48:427-35.

34. Biffi R, Luca F, Bianchi PP, Cenciarelli S, Petz W, Monsellato I, et al. Dealing with robot-assisted surgery for rectal cancer: current status and perspectives. World J Gastroenterol. 2016;22:546-56. 
35. Pai A, Marecik SJ, Park JJ, Melich G, Sulo S, Prasad LM. Oncologic and clinicopathologic outcomes of robot-assisted total mesorectal excision for rectal cancer. Dis Colon Rectum. 2015;58:659-67.

36. Kim CN, Bae SU, Lee SG, Yang SH, Hyun IG, Jang JH, et al. Clinical and oncologic outcomes of totally robotic total mesorectal excision for rectal cancer: initial results in a center for minimally invasive surgery. Int J Color Dis. 2016;31:843-52.

37. Feroci F, Vannucchi A, Bianchi PP, Cantafio S, Garzi A, Formisano G, et al. Total mesorectal excision for mid and low rectal cancer: laparoscopic vs robotic surgery. World J Gastroenterol. 2016;22:3602-10.

38. Cho MS, Baek SJ, Hur H, Min BS, Baik SH, Lee KY, et al. Short and long-term outcomes of robotic versus laparoscopic total mesorectal excision for rectal cancer: a case-matched retrospective study. Medicine (Baltimore). 2015;94:e522.

39. Yamaguchi T, Kinugasa Y, Shiomi A, Tomioka H, Kagawa H, Yamakawa Y. Robotic-assisted vs. conventional laparoscopic surgery for rectal cancer: short-term outcomes at a single center. Surg Today. 2016;46:957-62.

40. Park EJ, Cho MS, Baek SJ, Hur H, Min BS, Baik SH, et al. Long-term oncologic outcomes of robotic low anterior resection for rectal cancer: a comparative study with laparoscopic surgery. Ann Surg. 2015;261:129-37.

41. Ghezzi TL, Luca F, Valvo M, Corleta OC, Zuccaro M, Cenciarelli S, et al, Robotic versus open total mesorectal excision for rectal cancer: comparative study of short and long-term outcomes. Eur J Surg Onco. 2014;40:1072-9.

42. Ramji KM, Cleghorn MC, Josse JM, MacNeill A, O'Brien C, Urbach D, et al. Comparison of clinical and economic outcomes between robotic, laparoscopic, and open rectal cancer surgery: early experience at a tertiary care center. Surg Endosc. 2016;30:1337-43.

43. Hara M, Sng K, Yoo BE, Shin JW, Lee DW, Kim SH. Robotic-assisted surgery for rectal adenocarcinoma: short-term and midterm outcomes from 200 consecutive cases at a single institution. Dis Colon Rectum. 2014;57:570-7.

44. Baik SH, Kim NK, Lim DR, Hur H, Min BS, Lee KY. Oncologic outcomes and perioperative clinicopathologic results after robot-assisted tumor-specific mesorectal excision for rectal cancer. Ann Surg Oncol. 2013;20:2625-32.

\section{Submit your next manuscript to BioMed Central and we will help you at every step:}

- We accept pre-submission inquiries

- Our selector tool helps you to find the most relevant journal

- We provide round the clock customer support

- Convenient online submission

- Thorough peer review

- Inclusion in PubMed and all major indexing services

- Maximum visibility for your research

Submit your manuscript at www.biomedcentral.com/submit

) Biomed Central 